\title{
Quantum exact non-abelian vortices in non-relativistic theories
}

\author{
Muneto Nitta, ${ }^{a}$ Shun Uchino ${ }^{b}$ and Walter Vinci $^{c}$ \\ ${ }^{a}$ Department of Physics, and Research and Education Center for Natural Sciences, \\ Keio University, Hiyoshi 4-1-1, Yokohama, Kanagawa 223-8521, Japan \\ ${ }^{b} D P M C-M a N E P$, University of Geneva, \\ 24 Quai Ernest-Ansermet, CH-1211 Geneva, Switzerland \\ ${ }^{c}$ London Centre for Nanotechnology and Computer Science, \\ University College London, 17-19 Gordon Street, London, WC1H 0AH, U.K. \\ E-mail: nitta@phys-h.keio.ac.jp, shun.uchino@gmail.com, \\ wltvinci@gmail.com
}

ABSTRACT: Non-Abelian vortices arise when a non-Abelian global symmetry is exact in the ground state but spontaneously broken in the vicinity of their cores. In this case, there appear (non-Abelian) Nambu-Goldstone (NG) modes confined and propagating along the vortex. In relativistic theories, the Coleman-Mermin-Wagner theorem forbids the existence of a spontaneous symmetry breaking, or a long-range order, in $1+1$ dimensions: quantum corrections restore the symmetry along the vortex and the NG modes acquire a mass gap. We show that in non-relativistic theories NG modes with quadratic dispersion relation confined on a vortex can remain gapless at quantum level. We provide a concrete and experimentally realizable example of a three-component Bose-Einstein condensate with $\mathrm{U}(1) \times \mathrm{U}(2)$ symmetry. We first show, at the classical level, the existence of $S^{3} \simeq S^{1} \ltimes S^{2}$ $\left(S^{1}\right.$ fibered over $\left.S^{2}\right)$ NG modes associated to the breaking $\mathrm{U}(2) \rightarrow \mathrm{U}(1)$ on vortices, where $S^{1}$ and $S^{2}$ correspond to type I and II NG modes, respectively. We then show, by using a Bethe ansatz technique, that the $\mathrm{U}(1)$ symmetry is restored, while the $\mathrm{SU}(2)$ symmery remains broken non-pertubatively at quantum level. Accordingly, the U(1) NG mode turns into a $c=1$ conformal field theory, the Tomonaga-Luttinger liquid, while the $S^{2}$ NG mode remains gapless, describing a ferromagnetic liquid. This allows the vortex to be genuinely non-Abelian at quantum level.

Keywords: Solitons Monopoles and Instantons, Spontaneous Symmetry Breaking, Sigma Models, Bethe Ansatz

ARXIV EPRINT: 1311.5408 


\section{Contents}

1 Introduction 1

2 Effective Lagrangian approach $\quad 2$

3 Quantum exact gapless modes $\quad 6$

$\begin{array}{lll}4 & \text { Summary and discussion } & 7\end{array}$

$\begin{array}{ll}\text { A Derivation of the effective action } & 9\end{array}$

$\begin{array}{ll}\text { B Derivation of Bethe ansatz equations } & 9\end{array}$

$\begin{array}{ll}\text { C Derivation of dressed energies } & 12\end{array}$

D Tomonaga-Luttinger liquid in two-component Bose gases 13

\section{Introduction}

Vortices play a prominent role in various areas of physics such as superfluidity, superconductivity, magnetic materials, nematic liquids, fractional quantum Hall effect, topological quantum computation, quantum field theory and cosmology. In particular in high energy physics, much attention has been recently paid to non-Abelian vortices. Such objects arise when a non-Abelian symmetry is spontaneously broken in the vicinity of the vortex. The gapless Nambu-Goldstone (NG) modes associated to this symmetry breaking are then confined on the vortex, where they can propagate.

Non-Abelian vortices were first discovered in supersymmetric gauge theories [1-5] (see refs. [5-8] for a review), where their presence elegantly explains a long standing problem about quantum physics in different dimensions, i.e. similarities in the non-perturbative dynamics of sigma models in $1+1$ dimensions and Yang-Mills theory in $3+1$ dimensions (e.g. existence of dynamical mass gap, asymptotic freedom, instantons). Non-Abelian vortices also appear in high density quark matter (in this context also known as color flux tubes) [913] (see ref. [14] for a review). In this case, a Coleman-Weinberg quantum potential arising on the vortex world-sheet leads to the presence of monopole mesons confined by color flux tubes $[15,16]$. This phenomenon explains a quark-hadron duality between low and high density QCD, i.e. quarks are confined when monopoles are condensed at low density while monopoles are confined when quarks are condensed at high density [16]. The examples mentioned above correspond to relativistic systems. In such cases NG modes acquire mass gaps through quantum corrections $[17,18]$ consistently with the Coleman-Mermin-Wagner theorem $[19,20]$ which forbids the existence of a spontaneous symmetry breaking or a 
long-range order in $1+1$ dimensions. In this sense, non-Abelian vortices studied thus far in relativistic theories are Abelianized at quantum level [17, 21].

On the other hand, the situation can be different in non-relativistic theories. There are two kinds of NG modes, type-I and II, with linear and quadratic dispersions respectively [22]. This distinction is deeply connected with the geometry of the space parametrized by the NG modes [23, 24]; one type-II NG mode corresponds to two broken generators, while one type-I NG mode corresponds to one broken generator. In the relativistic case only type-I NG are allowed.

In this paper, we show that type-II NG modes confined in the core of a vortex remain gapless at quantum level, providing a quantum exact non-Abelian vortex. We consider a concrete example of a three-component Bose-Einstein condensate $(B E C)$ with $\mathrm{U}(2) \times \mathrm{U}(1)$ symmetry, in which there is partial phase separation between one (immiscible) component and the other two (miscible) components. Our setup can be realized in experiments of ultracold atomic gases; (Almost) U(2) symmetric two-component BECs were already realized by ultracold ${ }^{87} \mathrm{Rb}[25]$ and ${ }^{23} \mathrm{Na}[26]$ atoms. If one mixes additional atom with repulsive interaction with these atoms, one can experimentally realize our set-up. In this system, we find that there appear an $S^{1}$ type-I NG mode and an $S^{2} \simeq \mathbb{C} P^{1}$ type-II NG mode in a vortex core. By noting that this vortex acts as a $1+1$ dimensional trap for the two miscible components, we describe the modes living in the vortex core as a $1+1$ dimensional two-component Bose gas with U(2) symmetry. By studying the exact solution of this system through a Bethe ansatz technique, we show that at quantum level the U(1) symmetry recovers and the $\mathrm{U}(1)$ sector can be described by a conformal field theory (CFT) with the conformal charge $c=1$ or a Tomonaga-Luttinger liquid [27, 28]. On the other hand, the $\mathrm{SU}(2)$ symmetry breaking holds at quantum level and the $S^{2} \mathrm{NG}$ mode remains gapless, describing a ferromagnetic liquid [29].

\section{Effective Lagrangian approach}

We consider a three-component BEC with $\mathrm{U}(1) \times \mathrm{U}(2)$ symmetry. The Lagrangian for the Gross-Pitaevskii equation is

$$
\begin{aligned}
\mathcal{L}= & i \hbar \psi_{0}^{\dagger} \dot{\psi}_{0}+i \hbar \Psi^{\dagger} \dot{\Psi}-\frac{\hbar^{2}}{2 m_{0}} \nabla_{i} \psi_{0}^{\dagger} \nabla_{i} \psi_{0}-\frac{\hbar^{2}}{2 m} \nabla_{i} \Psi^{\dagger} \nabla_{i} \Psi \\
& +\mu_{0}\left|\psi_{0}\right|^{2}+\mu|\Psi|^{2}-\frac{1}{2} \lambda_{0}\left|\psi_{0}\right|^{4}-\frac{1}{2} \lambda|\Psi|^{4}-\kappa\left|\psi_{0}\right|^{2}|\Psi|^{2},
\end{aligned}
$$

which is written in terms of the condensate wave functions $\left(\psi_{0}, \Psi^{T}\right)$, where $\Psi$ is a twocomponent condensate. Here, $\mu$ and $\mu_{0}$ are chemical potentials, and $\lambda\left(\lambda_{0}\right)$ and $\kappa$ are intracomponent and intercomponent couplings, respectively. The system above has a stable minimum when $\lambda_{0} \lambda-\kappa^{2}>0$. We consider the phase separation between $\psi_{0}$ (immiscible component) and $\Psi$ (miscible components), with a non-vanishing expectation value for $\psi_{0}$, which is obtained when $\mu_{0} \lambda-\mu \kappa>0, \mu \lambda_{0}-\mu_{0} \kappa<0$ and $\mu^{2} \lambda_{0}-\mu_{0}^{2} \lambda<0$. In this situation, the ground state is $\left(\psi_{0}, \Psi^{T}\right)=(v, 0,0)$ with $v^{2}=\mu_{0} / \lambda_{0}$, where the symmetry is spontaneously broken down to $\mathrm{U}(2)$. 
In the cylindrical coordinates $(r, \theta, z)$, a vortex along the $z$-axis is given by

$$
\left(\begin{array}{c}
\psi_{0} \\
\Psi
\end{array}\right)=\left(\begin{array}{c}
f_{0}(r, \theta) e^{i \theta} \\
g_{0}(r, \theta) \eta
\end{array}\right), \quad \eta^{\dagger} \eta=1,
$$

with the boundary conditions $f_{0} \rightarrow v$ and $g_{0} \rightarrow 0$ at $r \rightarrow \infty$ and $f_{0} \rightarrow 0$ and $g_{0}^{\prime} \rightarrow 0$ at $r \rightarrow 0$, see figure 1. Here, $\eta$ is a two-component complex constant that parameterizes $S^{3}$, and identify the NG modes associated with the ground state symmetry breaking $\mathrm{U}(2) \rightarrow$ $\mathrm{U}(1)$ that takes place in the vicinity of the vortex core: $\mathrm{U}(2) / \mathrm{U}(1) \simeq \mathrm{SU}(2) \simeq S^{3}$.

We construct a low-energy effective theory of the vortex through the moduli approximation $[30,31]$ introducing a world-sheet coordinate $(t, z)$ dependence of the vortex configuration

$$
\left(\begin{array}{c}
\psi_{0} \\
\Psi
\end{array}\right)=\left(\begin{array}{c}
f(r, \theta, z, t) e^{i \theta} \\
g(r, \theta, z, t) \eta(z, t)
\end{array}\right), \quad \eta^{\dagger} \eta=1 .
$$

Notice that we have included a world-sheet coordinate dependence on the profile functions too. This is equivalent to the inclusion of "Higgs" excitations $H$ and $h$ in the calculation:

$$
\begin{aligned}
& f(r, \theta, z, t) \equiv f_{0}(r)+H(r, \theta, z, t), \\
& g(r, \theta, z, t) \equiv g_{0}(r)+h(r, \theta, z, t) .
\end{aligned}
$$

This modification to the standard approach in deriving effective theories in the relativistic case is crucial. The field $H$ is a massive bulk mode, while the field $h$ is a lighter nonpropagating mode that contributes to the effective action for the zero-modes $\eta$.

By substituting eq. (2.3) in the Lagrangian we get:

$$
\begin{aligned}
\mathcal{L}= & i \hbar g^{2} \eta^{\dagger} \dot{\eta} \\
& -\frac{\hbar^{2}}{2 m_{0}}\left[f^{\prime 2}+\frac{1}{r^{2}} f^{2}+\frac{1}{r^{2}}\left(\nabla_{\theta} f\right)^{2}+\left(\nabla_{z} f\right)^{2}\right] \\
& -\frac{\hbar^{2}}{2 m}\left[g^{\prime 2}+\frac{1}{r^{2}}\left(\nabla_{\theta} g\right)^{2}+\left(\nabla_{z} g\right)^{2}+g^{2}\left|\nabla_{z} \eta\right|^{2}\right] \\
& +\mu_{0} f^{2}+\mu g^{2}-\frac{1}{2} \lambda_{0} f^{4}-\frac{1}{2} \lambda g^{4}-\kappa f^{2} g^{2},
\end{aligned}
$$

from whose variation one obtains the equations of motion for $f$ and $g$ :

$$
\begin{aligned}
& 0=-\frac{\hbar^{2}}{2 m_{0}}\left[\frac{1}{r^{2}} f-\triangle f\right]+\mu_{0} f-\lambda_{0} f^{3}-\kappa f g^{2} \\
& 0=i \hbar g \eta^{\dagger} \dot{\eta}-\frac{\hbar^{2}}{2 m}\left[g\left|\nabla_{z} \eta\right|^{2}-\triangle g\right]+\mu g-\lambda g^{3}-\kappa g f^{2} .
\end{aligned}
$$

In the derivation of the effective action for the moduli $\eta$ we will keep only terms up to second derivatives in the world-sheet coordinates. This allows us to consider an expansion of the equations above in powers of these coordinates. An important observation is that, thanks to the (static) equation of motions, the corrections to the effective Lagrangian in eq. (2.5) enter quadratically at least. This allows us to calculate all terms in the effective action, 


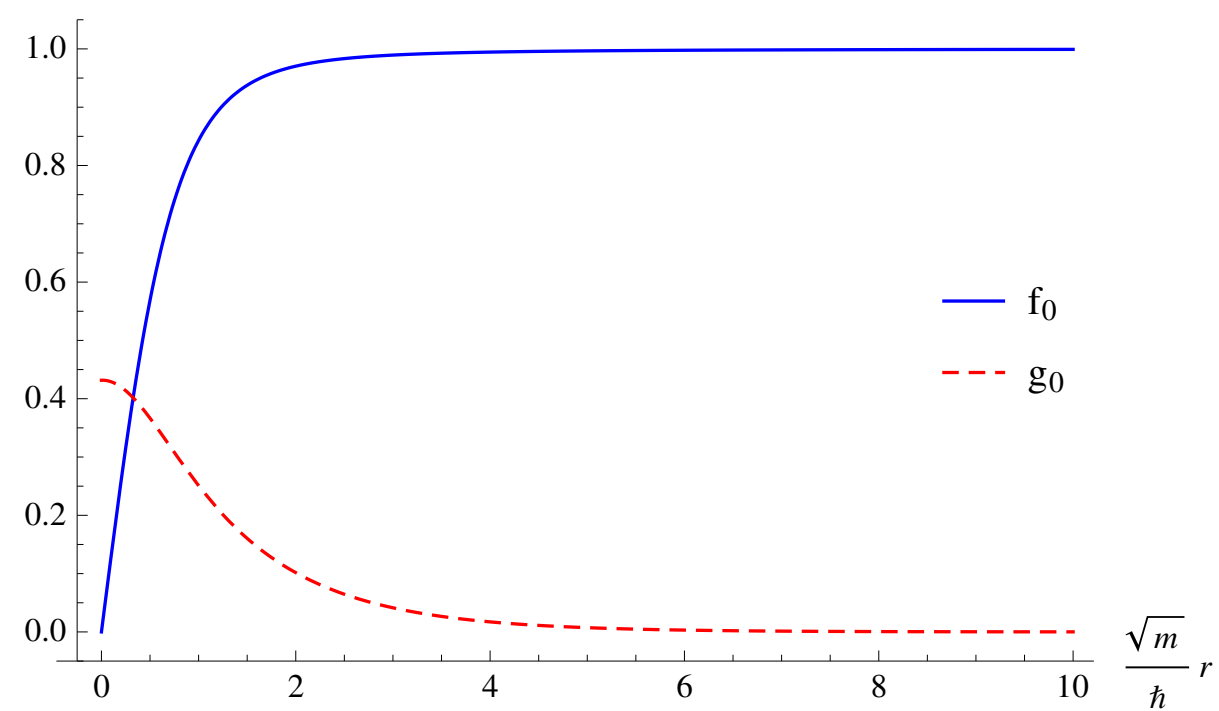

Figure 1. Numerical solution for the profile functions $f_{0}$ and $g_{0} . \mu=2, \mu_{0}=\lambda=\lambda_{0}=3, \kappa=2.3$, $m=m_{0}$.

up the the second order in the world-sheet coordinates, by just calculating corrections to the profile functions up to the first order. As can be seen by inspecting eq. (2.6), first order corrections will only involve time derivatives of the moduli $\eta$. We proceed using the following expansion:

$$
\begin{aligned}
& g=g_{0}+i \hbar \eta^{\dagger} \dot{\eta} g_{1}+\mathcal{O}\left(\partial_{t}^{2}, \partial_{z}^{2}\right), \\
& f=f_{0}+i \hbar \eta^{\dagger} \dot{\eta} f_{1}+\mathcal{O}\left(\partial_{t}^{2}, \partial_{z}^{2}\right)
\end{aligned}
$$

We first consider the zeroth order, corresponding to the static case, and study the vortex profile functions $f_{0}$ and $g_{0}$. The equations for these two functions correspond to those in eq. (2.6) without the world-sheet derivatives. A numerical example can be seen in figure 1. We can see how the vortex built in the immiscible component acts as a one dimensional trap for the two miscible components. This is the first crucial observation of our paper. The asymptotic behavior of the profile functions can be found analytically by studying the expansion $f_{0}=v-\delta f, g_{0}=\delta g$ of eq. (2.6) from which we get:

$$
\begin{aligned}
& f_{0} \sim v-\frac{1}{2 \sqrt{\mu_{0} \lambda_{0}}} \frac{1}{r^{2}}, \\
& g_{0} \sim \frac{c_{1}}{\sqrt{r}} e^{-\gamma r}, \quad \gamma \equiv \sqrt{\frac{-\lambda_{0} \mu+\kappa \mu_{0}}{\lambda_{0}}} .
\end{aligned}
$$

As common in systems with only global symmetries, the profile function $f_{0}$ for the condensate supporting the winding decays polynomially. On the other hand the expression above shows the profile function for the trapped components $g_{0}$ decays exponentially. This is our second important observation. The exponential decay allows us to search for normalizable zero-modes propagating on the vortex line, so that the $1+1$ effective action is finite on an infinite volume. 
We proceed now to the calculation of the effective action with the derivation of the higher order corrections $f_{1}$ and $g_{1}$. The expansion of eq. (2.6) in powers of the worldsheet coordinates gives new differential linear equations for the variables $f_{1}$ and $g_{1}$ (see appendix A). Since these equations are linear in the first order corrections, $f_{1}(r)$ end $g_{1}(r)$ depend only on the zeroth order profile functions $f_{0}$ and $g_{0}$. Their numerical evaluation can be found in figure 2 in appendix A. Substituting the expressions $g=g_{0}+i \hbar \eta^{\dagger} \dot{\eta} g_{1}$ and $f=f_{0}+i \hbar \eta^{\dagger} \dot{\eta} f_{1}$ in the original Lagrangian (2.5), and remembering to keep track of the second order terms only, we obtain:

$$
\begin{aligned}
\mathcal{L} & =i \hbar g_{0}^{2} \eta^{\dagger} \dot{\eta}+K \hbar^{2}\left(\eta^{\dagger} \dot{\eta}\right)^{2}-\frac{\hbar^{2}}{2 m} g_{0}^{2}\left|\nabla_{z} \eta\right|^{2} \\
K & \equiv-\left(\mu_{0} f_{1}^{2}+\mu g_{1}^{2}-3 \lambda_{0} f_{0} f_{1}^{2}-3 \lambda g_{0} g_{1}^{2}-4 \kappa f_{0} g_{0} f_{1} g_{1}-\kappa g_{0}^{2} f_{1}^{2}-\kappa f_{0}^{2} g_{1}^{2}\right)
\end{aligned}
$$

The effective action is obtained after integrating over the transverse direction $r$, this can be easily done numerically with the knowledge of the profile functions $f_{0,1}$ and $g_{0,1}$. The whole calculation has assumed only a derivative expansion in powers of the world-sheet derivatives, keeping terms up to the second order:

$$
\begin{aligned}
\mathcal{L}_{\text {eff }} & =\alpha i \hbar \eta^{\dagger} \dot{\eta}-\alpha \frac{\hbar^{2}}{2 m}\left|\nabla_{z} \eta\right|^{2}-\beta \hbar^{2}\left(\eta^{\dagger} \dot{\eta}\right)^{2} \\
\alpha & =\int_{0}^{\infty} 2 \pi r d r g_{0}^{2}, \quad \beta=\int_{0}^{\infty} 2 \pi r d r K .
\end{aligned}
$$

Since the function $g_{0}$ is exponentially decaying, the coefficient $\alpha$ is finite. Moreover, notice that the first order correction $i \hbar g_{0} \eta^{\dagger} \dot{\eta}$ is exponentially small at large distances, which implies that also the functions $f_{1}$ and $g_{1}$ decay exponentially. As a consequence, the coefficient $\beta$ is finite and of the same order of $\alpha$. This means that the effective action is finite and the zero modes are truly confined on the vortex. The coefficients $\alpha$ and $\beta$ have been calculated numerically in appendix A for the special choice of parameters shown in figure 1.

In order to see the dispersions of the NG modes, we recall that the geometry of the NG modes is $S^{3} \simeq S^{1} \ltimes S^{2}$, where $S^{1}$ is fibered over $S^{2} \simeq \mathbb{C} P^{1}$. To make this structure explicit, we take a parameterization

$$
\eta=e^{i \varphi} n
$$

with $n^{\dagger} n=1$, where $\varphi$ and $n$ parametrize $S^{1}$ and $S^{2}$. Then, the effective Lagrangian can be rewritten as

$$
\begin{aligned}
\mathcal{L}_{\mathrm{eff} .}= & -\beta \hbar^{2}\left(\dot{\varphi}-\frac{i}{2}\left(n^{\dagger} \dot{n}-\dot{n}^{\dagger} n\right)\right)^{2} \\
& -\alpha \frac{\hbar^{2}}{2 m}\left(\partial_{z} \varphi-\frac{i}{2}\left(n^{\dagger} \partial_{z} n-\partial_{z} n^{\dagger} n\right)\right)^{2} \\
& +2 i \alpha \hbar\left(n^{\dagger} \dot{n}-\dot{n}^{\dagger} n\right)-\alpha \frac{\hbar^{2}}{2 m}\left(\left(\partial_{z} n\right)^{2}-\left(n^{\dagger} \partial_{z} n\right)^{2}\right),
\end{aligned}
$$


which shows that $\varphi$ and $n$ are type-I and II NG modes with linear and quadratic dispersions, respectively. Here, remarkably $n$ describes one type-II NG mode corresponding to the two broken generators of $S^{2}$. In addition, there appears one type-II translational zero mode corresponding to two broken translational symmetries [32].

\section{Quantum exact gapless modes}

In the effective theory approach, we have obtained gapless modes at classical level. In order to discuss whether these modes remain gapless quantum mechanically, we should take into account massive modes and quartic interaction. As we have noticed, the vortex in the first component can be seen as a trap for the second and third components, resulting in two-component Bose gases with $\mathrm{U}(2)$ symmetry in $1+1$ dimension. Here, we study such two-component Bose gases at quantum level.

Two-component Bose gases with U(2) symmetry in $1+1$ dimension are also known as Yang-Gaudin model [33, 34]. Including a nonlinear interaction, the Hamiltonian in units of $\hbar^{2} /(2 m)$ is given by

$$
H=\int_{0}^{L} d z\left(\sum_{\sigma=1,2} \partial_{z} \Psi_{\sigma}^{\dagger} \partial_{z} \Psi_{\sigma}-\mu \rho+\lambda \rho^{2}\right),
$$

where $\rho=\sum_{\sigma} \Psi_{\sigma}^{\dagger} \Psi_{\sigma}$ is the density, and $L$ is a system size along the $z$-direction. The effective parameters $\lambda$ and $\mu$ can be related to the original parameters through a renormalization group, but here we do not need explicit relations. Since there is no spin-dependent coupling, the Hamiltonian has U(2) symmetry in which we can use the Bethe ansatz technique $[28,35]$. The resultant Bethe ansatz equations are [36, 37] (See appendix B)

$$
\begin{aligned}
k_{j} L & =2 \pi I_{j}-\sum_{l=1}^{N} \Theta\left(\frac{k_{j}-k_{l}}{\lambda}\right)+\sum_{\beta=1}^{M} \Theta\left(\frac{2 k_{j}-2 l_{\beta}}{\lambda}\right) \\
2 \pi J_{\alpha} & =\sum_{j=1}^{N} \Theta\left(\frac{2 l_{\alpha}-2 k_{j}}{\lambda}\right)-\sum_{\beta=1}^{M} \Theta\left(\frac{l_{\alpha}-l_{\beta}}{\lambda}\right)
\end{aligned}
$$

where $\Theta(x)=2 \tan ^{-1}(x)$, and $N$ and $M$ represent the total number of the particles and particle number for the component " 2 ", respectively. $k_{j}$ and $l_{\alpha}$ are called quasimomenta and isospin rapidities originating from $\mathrm{SU}(2)$ symmetry in the system. $I_{j}$ and $J_{\alpha}$ take integer or half-integer values, depending on whether $N-M$ is odd or even. Then, the total energy and momentum are given by

$$
E=\sum_{j=i}^{N}\left(k_{j}^{2}-\mu\right), \quad P=\sum_{j=1}^{N} k_{j} .
$$

By using $I_{j}$ and $J_{\alpha}$, the ground state can be characterized as [36]

$$
\left\{I_{j}\right\}=\{-(N-1) / 2, \cdots,(N-1) / 2\}, \quad M=0 .
$$


In the thermodynamic limit, the above Bethe ansatz equations are then reduced to

$$
\rho(k)=\frac{1}{2 \pi}+\frac{1}{\pi} \int_{-\Lambda}^{\Lambda} d k^{\prime} \frac{\lambda \rho\left(k^{\prime}\right)}{\lambda^{2}+\left(k-k^{\prime}\right)^{2}},
$$

where $\rho(k)$ is the density of quasimomenta, and cut-off $\Lambda$ is determined by the density of the system $N / L=\int_{-\Lambda}^{\Lambda} \rho(k)$. This equation corresponds to that in Lieb-Liniger gas [38, 39]. Thus, as far as $S^{1}$ sector is concerned, the low-energy excitation is linear gapless characterized by the $c=1 \mathrm{CFT}$ while there is no BEC. In addition, $M=0$ means that the ground state is ferromagnetic, which is consistent with a general theorem that a ground state of spinful bosons without spin-dependent interaction is always polarized [40] .

We next consider the nature of the isospin excitation originating from $S^{2}$ manifold. Although analytic results can be obtained for weak and strong coupling limits, we need to numerically determine it for general couplings. To this end, we consider a dressed energy formalism in the Bethe ansatz [28]. In our model, we can obtain

$$
\begin{aligned}
& \epsilon_{c}(k)=k^{2}-\mu+\frac{1}{\pi} \int_{-\Lambda}^{\Lambda} d k^{\prime} \frac{\lambda \epsilon_{c}\left(k^{\prime}\right)}{\lambda^{2}+\left(k-k^{\prime}\right)^{2}} \\
& \epsilon_{s}(l)=-\frac{1}{2 \pi} \int_{-\Lambda}^{\Lambda} d k^{\prime} \frac{\lambda \epsilon_{c}\left(k^{\prime}\right)}{(\lambda / 2)^{2}+\left(l-k^{\prime}\right)^{2}}
\end{aligned}
$$

where $\mu$ is the chemical potential and we introduced the dressed energy $\epsilon_{c}(k)$ and $\epsilon_{s}(l)$, each of which represents $S^{1}$ and $S^{2}$ excitations. The derivation of the above equations is given in appendix B. In the low-energy isospin excitation, an isospin rapidity $l$ is related to the real momentum of the isospin excitation $p$ as follows:

$$
p=\int_{-\Lambda}^{\Lambda} d k^{\prime} \rho\left(k^{\prime}\right)\left[\Theta\left(\frac{2 k^{\prime}-2 l}{\lambda}\right)+\pi\right]
$$

By solving a couple of the above equations, we can confirm that the isospin excitation is gapless quadratic one [36, 37]. This indicates that a type-II NG mode, called a magnon, emerges for the isospin sector due to a spontaneous symmetry breaking of SU(2).

\section{Summary and discussion}

We have shown that a non-Abelian vortex remains non-Abelian at the quantum level when trapped NG modes are of type-II, e.g. the low-energy behavior of the NG modes is robust against quantum fluctuations. We have worked out an explicit example of a three component BEC with $\mathrm{U}(2) \times \mathrm{U}(1)$ symmetry. At the classical level, there appear in the $1+1$ dimensional low-energy effective theory of the vortex both type I and II NG modes. $\varphi$ and $n$, with linear and quadratic dispersions respectively, are associated to the fiber $S^{1}$ and the base $S^{2}$ of the moduli space $S^{3} \simeq S^{1} \ltimes S^{2}$, where $S^{3}$ is associated with the symmetry breaking induced by a vortex solution $\mathrm{U}(2) \rightarrow \mathrm{U}(1)$. At quantum level, however, the $\mathrm{U}(1)$ symmetry is recovered while $\varphi$ remains gapless with a linear dispersion. On the other hand, the symmetry breaking $\mathrm{SU}(2) \rightarrow \mathrm{U}(1)$ holds at quantum level and $n$ remains gapless with a quadratic dispersion. We then should interpret the type-I NG mode $\varphi$ as the $c=1$ CFT 
mode at quantum level since there is no BEC, that is, a Tomonaga-Luttinger liquid. At the same time, the type-II NG mode $n$ remains at quantum level and is interpreted as a ferromagnetic liquid.

An intuitive understanding of the above is as follows. As far as the low-energy physics is concerned where $S^{1}$ and isospin sectors are expected to be separated, the kinetic Hamiltonians in $S^{1}$ and isospin sectors are Lorentz and Galilei invariant, respectively, thanks to their dispersion rules. Then, while the spontaneous symmetry breaking in the $S^{1}$ sector is forbidden by the Coleman-Mermin-Wagner theorem, there still exists a linear gapless mode since the fixed point of the system is the so-called Tomogana-Luttinger liquid where all of the interactions that can yield gaps in the system are irrelevant. On the other hand, the spin sector is purely non-relativistic in the sense that Lorentz symmetry does not emerge even in the low-energy physics. Since one of the conditions of the Coleman-Mermin-Wagner theorem is Lorentz invariance, spontaneous symmetry breaking in the isospin sector is not forbidden and the type-II NG mode remains at the quantum level as prescribed by the NG theorem.

We can also consider the case where the $\mathrm{U}(2)$ symmetry is explicitly broken to $\mathrm{U}(1)^{2}$, but the trapped components are still miscible. This can be obtained with an additional term of the type $-\mathcal{L}_{\text {int }}=\lambda_{12}\left|\Psi_{1}\right|^{2}\left|\Psi_{2}\right|^{2}+\frac{1}{2} \lambda_{11}\left|\Psi_{1}\right|^{4}+\frac{1}{2} \lambda_{22}\left|\Psi_{2}\right|^{4}$, where $\lambda_{12}$ is smaller than intracomponent couplings in $\lambda_{11}$ and $\lambda_{22}$ (the $\mathrm{U}(2)$ symmetric case corresponds to $\lambda_{11}=\lambda_{22}=\lambda_{12}=\lambda$ ).

At the classical level, this symmetry is spontaneously broken in the vortex core and two type-I NG modes are trapped in the vortex. At the quantum level, the $\mathrm{U}(1)^{2}$ symmetry is recovered and there are two Tomonaga-Luttinger liquids as far as the so-called TomonagaLuttinger parameter in the isospin sector is greater than 1 (See appendix B).

It is straightforward to extend our model to an $(N+1)$-component BEC with $\mathrm{U}(N) \times \mathrm{U}(1)$ symmetry. There appear NG modes $S^{N-1} \simeq S^{1} \ltimes \mathbb{C} P^{N-1}$, where $S^{1}$ and $\mathbb{C} P^{N-1}$ are type-I and II NG modes at the classical level. At the quantum level, the $\mathrm{U}(1)$ symmetry is recovered with the Tomonaga-Luttinger liquid, while the $\mathrm{SU}(N)$ symmetry remains broken and $\mathbb{C} P^{N-1}$ modes remain as type-II NG modes, as ensured by the Eisenberg-Lieb theorem [40].

Finally, fate of type-II NG modes localized on domain walls [41, 42] and skyrmion lines [43] in the presence of quantum effects is one interesting future problem to explore.

\section{Acknowledgments}

The work of MN is supported in part by Grant-in-Aid for Scientific Research (No. 25400268) and by the "Topological Quantum Phenomena" Grant-in-Aid for Scientific Research on Innovative Areas (No. 25103720) from the Ministry of Education, Culture, Sports, Science and Technology (MEXT) of Japan. SU is supported by the Swiss National Science Foundation under MaNEP and division II. The work of WV has been supported by a Global Engagement for Global Impact programme funded by EPSRC. 


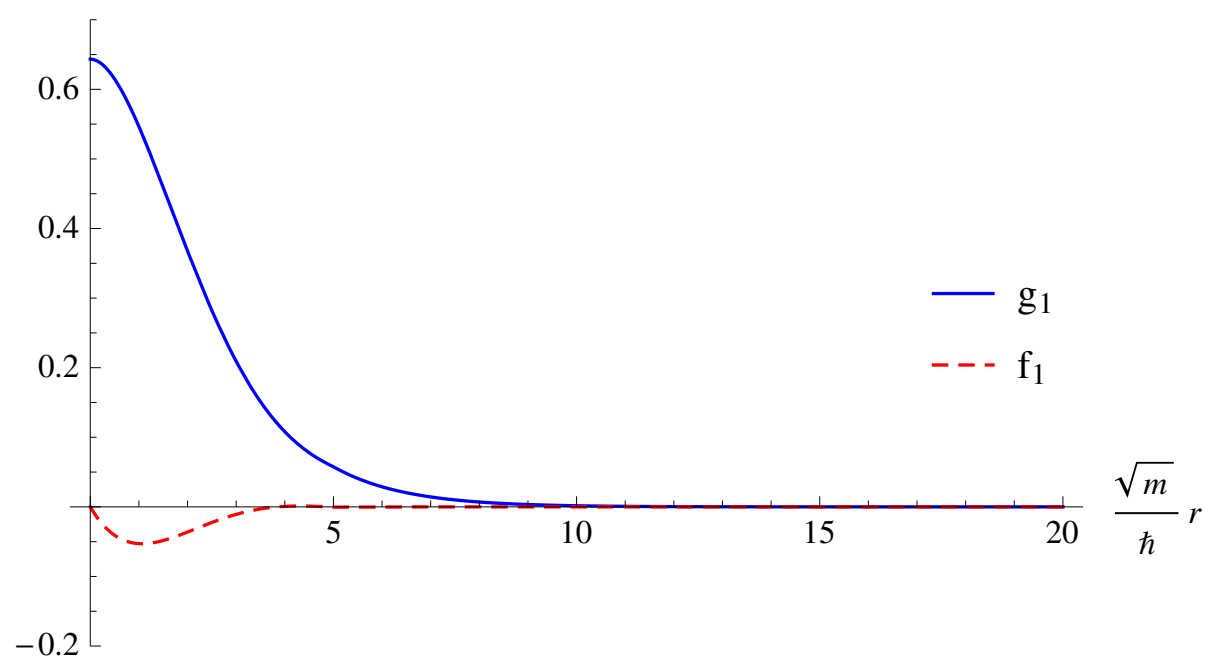

Figure 2. Numerical solution for the first order corrections to the profile functions $f_{0}$ and $g_{0}$. $\mu=2, \mu_{0}=\lambda=\lambda_{0}=3, \kappa=2.3, m=m_{0}$.

\section{A Derivation of the effective action}

Assuming the following derivative expansion for the profile functions as explained in the text

$$
\begin{aligned}
& g=g_{0}+i \hbar \eta^{\dagger} \dot{\eta} g_{1}+\mathcal{O}\left(\partial_{t}^{2}, \partial_{z}^{2}\right) \\
& f=f_{0}+i \hbar \eta^{\dagger} \dot{\eta} f_{1}+\mathcal{O}\left(\partial_{t}^{2}, \partial_{z}^{2}\right)
\end{aligned}
$$

we obtain the following equations:

$$
\begin{aligned}
0 & =-\frac{\hbar^{2}}{2 m_{0}}\left[\frac{1}{r^{2}} f_{1}-\triangle f_{1}\right]+\mu_{0} f_{1}-3 \lambda_{0} f_{0}^{2} f_{1}-\kappa f_{1} g_{0}^{2}-2 \kappa f_{0} g_{0} g_{1} \\
0 & =g_{0}-\frac{\hbar^{2}}{2 m}\left[-\triangle g_{1}\right]+\mu g_{1}-3 \lambda g_{0}^{2} g_{1}-\kappa g_{1} f_{0}^{2}-2 \kappa g_{0} f_{0} f_{1} .
\end{aligned}
$$

Since the non-homogeneous term in the effective action is exponentially small, the functions $f_{1}$ and $g_{1}$ are also exponentially small. A numerical solution for these quantities is shown in figure 2. Using eq. (2.10), we can evaluate numerically the coefficients $\alpha$ and $K$. Using the values of the parameters as indicated in the figures captions, we have, for example:

$$
\alpha=0.684, \quad \beta=1.09
$$

where we have taken $\hbar=m=1$.

\section{B Derivation of Bethe ansatz equations}

Here we sketch a derivation of the Bethe ansatz equations in two-component bosons, which can be solved by the so-called nested Bethe ansatz method. Our derivation is based on one in ref. [35], which discusses the Fermionic Hubbard model. 
We first consider $N$ particle wavefunction as

$$
\begin{aligned}
& \Psi\left(x_{1}, \cdots, x_{N} ; \sigma_{1}, \cdots, \sigma_{N}\right)=\sum_{\{P\}} A_{\sigma_{Q_{1}} \cdots \sigma_{Q_{N}}}\left(k_{p_{1}}, \cdots, k_{p_{N}}\right) e^{i \sum_{j=1}^{N} k_{p_{j}} x_{Q_{j}}}, \\
& 1 \leq x_{Q_{1}} \leq x_{Q_{2}} \cdots \leq x_{Q_{N}} \leq L
\end{aligned}
$$

where $\{P\}$ denotes possible permutations. From the continuity of the wavefunction and its first derivative at equal positions as $x_{1}=x_{2}$, we have

$$
\begin{aligned}
A_{\sigma_{Q_{1}} \cdots, \sigma_{Q_{i}}, \sigma_{Q_{i+1}}, \cdots \sigma_{Q_{N}}}\left(k_{p_{1}}, \cdots, k_{p_{i+1}}, k_{p_{i}}, \cdots, k_{p_{N}}\right) & \\
= & \frac{-i \lambda}{k_{p_{i}}-k_{p_{i+1}}+i \lambda} A_{\sigma_{Q_{1}} \cdots, \sigma_{Q_{i}}, \sigma_{Q_{i+1}}, \cdots \sigma_{Q_{N}}}\left(k_{p_{1}}, \cdots, k_{p_{i}}, k_{p_{i+1}}, \cdots, k_{p_{N}}\right) \\
& \quad+\frac{k_{p_{i}}-k_{p_{i+1}}}{k_{p_{i}}-k_{p_{i+1}}+i \lambda} A_{\sigma_{Q_{1}} \cdots, \sigma_{Q_{i+1}}, \sigma_{Q_{i}}, \cdots \sigma_{Q_{N}}}\left(k_{p_{1}}, \cdots, k_{p_{i}}, k_{p_{i+1}}, \cdots, k_{p_{N}}\right) .
\end{aligned}
$$

We next introduce

$$
\left|k_{p_{1}}, \cdots, k_{p_{N}}\right\rangle=\sum_{\left\{\sigma_{i}\right\}=\uparrow, \downarrow} A_{\sigma_{Q_{1}}, \cdots \sigma_{Q_{N}}}\left(k_{p_{1}}, \cdots, k_{p_{N}}\right)\left|\sigma_{1}, \cdots, \sigma_{N}\right\rangle,
$$

and

$$
\begin{aligned}
Y^{(a, b)}(x) & =\frac{-i \lambda}{x+i \lambda} I+\frac{x}{x+i \lambda} \Pi^{(a, b)} \\
\left|\sigma_{1}, \cdots, \sigma_{b}, \sigma_{a}, \cdots, \sigma_{N}\right\rangle & =\Pi^{(a, b)}\left|\sigma_{1}, \cdots, \sigma_{a}, \sigma_{b}, \cdots, \sigma_{N}\right\rangle
\end{aligned}
$$

with the identify operator $I$. Then, eq. (B.3) can be expressed as

$$
Y^{(a, b)}\left(k_{p_{i}}-k_{p_{i+1}}\right)\left|k_{p_{1}}, \cdots, k_{p_{i}}, k_{p_{i+1}} \cdots, k_{p_{N}}\right\rangle=\left|k_{p_{1}}, \cdots, k_{p_{i+1}}, k_{p_{i}} \cdots, k_{p_{N}}\right\rangle .
$$

We next consider the periodic boundary condition: $\Psi\left(x_{1}, \cdots, x_{i-1}, 1, x_{i+1}, \cdots, x_{N}\right)=$ $\Psi\left(x_{1}, \cdots, x_{i-1}, L+1, x_{i+1}, \cdots, x_{N}\right)$. Then,

$$
\begin{array}{r}
A_{\sigma_{Q_{1}} \cdots \sigma_{Q_{N}}}\left(k_{p_{1}}, \cdots, k_{p_{N}}\right)= \\
e^{i p_{1} L} A_{\sigma_{Q_{2}} \cdots \sigma_{Q_{N}}, \sigma_{Q_{1}}}\left(k_{p_{2}}, \cdots, k_{p_{N}}, k_{p_{1}}\right) .
\end{array}
$$

By substituting the above into eq. (B.4), we have

$$
\begin{aligned}
\left|k_{p_{1}}, \cdots, k_{p_{N}}\right\rangle & =e^{i p_{1} L} \prod_{j=0}^{N-2} X^{(1, N-j)}\left(k_{p_{1}}-k_{p_{N-j}}\right)\left|k_{p_{1}}, \cdots, k_{p_{N}}\right\rangle, \\
X^{(j, k)}(x) & =\Pi^{(j, k)} Y^{(j, k)}(x) .
\end{aligned}
$$

By introducing

$$
\begin{aligned}
S & \equiv \prod_{j=1}^{N-1} X^{(a, N-J)}\left(k_{p_{1}}-k_{p_{N-j}}\right) \\
& =-X^{(a, N)}\left(k_{p_{1}}-k_{p_{N}}\right) \cdots X^{(a, 2)}\left(k_{p_{1}}-k_{p_{2}}\right) \Pi^{(a, 1)}
\end{aligned}
$$


which satisfies

$$
\operatorname{Tr}_{a} S=-\prod_{j=0}^{N-2} X^{(1, N-j)}\left(k_{p_{1}}-k_{p_{N-j}}\right)
$$

we have

$$
\left|k_{p_{1}} \cdots k_{p_{N}}\right\rangle=-e^{i k_{p_{1}} L} \operatorname{Tr}_{a} S\left|k_{p_{1}} \cdots k_{p_{N}}\right\rangle .
$$

By using Pauli matrices, we have

$$
\begin{aligned}
X^{(a, j)}\left(l-l_{j}\right) & =\frac{1}{l-l_{j}+i \lambda}\left[-i \lambda \Pi^{(a, j)}+\left(l-l_{j}\right) I^{a} \otimes I^{(j)}\right] \\
& =\frac{1}{l-l_{j}+i \lambda}\left(\begin{array}{cc}
\left(l-l_{j}-i \lambda / 2\right) I_{j}-i \lambda \sigma_{j}^{z} / 2 & i \lambda \sigma_{j}^{-} \\
i \lambda \sigma_{j}^{+} & \left(l-l_{j}-i \lambda / 2\right) I_{j}+i \lambda \sigma_{j}^{z} / 2
\end{array}\right),
\end{aligned}
$$

with $l_{j}=k_{p_{j}}-i \lambda / 2$ since

$$
\Pi^{(a, j)}=\frac{1}{2}\left(\begin{array}{cc}
I_{j}+\sigma_{j}^{z} / 2 & 2 \sigma_{j}^{-} \\
2 \sigma_{j}^{+} & I_{j}-\sigma_{j}^{z} / 2
\end{array}\right) .
$$

We next introduce the so-called transfer matrix

$$
\begin{aligned}
T^{(a)}(l) & =X^{(a, N)}\left(l-l_{N}\right) \cdots X^{(a, 1)}\left(l-l_{1}\right) \\
& \equiv\left(\begin{array}{ll}
A(l) & B(l) \\
C(l) & D(l)
\end{array}\right) .
\end{aligned}
$$

By considering a "vacuum" state as $|\mathrm{vac}\rangle=|1, \cdots, 1\rangle$, we obtain

$$
\begin{aligned}
& A(l)|\mathrm{vac}\rangle=\prod_{j=1}^{N}\left(\frac{l-l_{j}-i \lambda}{l-l_{j}+i \lambda}\right)|\mathrm{vac}\rangle, \\
& C(l)|\mathrm{vac}\rangle=0, \\
& D(l)|\mathrm{vac}\rangle=\prod_{j=1}^{N}\left(\frac{l-l_{j}}{l-l_{j}+i \lambda}\right)|\mathrm{vac}\rangle .
\end{aligned}
$$

Since $Y^{(a, b)}$ and $T^{(a)}$ satisfy the celebrated Yang-Baxter relation

$$
Y^{(a, b)}(l-\mu) T^{(a)}(l) T^{(b)}(\mu)=T^{(a)}(\mu) T^{(b)}(l) Y^{(a, b)}(l-\mu),
$$

it is straightforward to show the following commutation relations:

$$
\begin{aligned}
B(l) B(\mu) & =B(\mu) B(l), \\
A(\mu) B(l) & =\frac{l-\mu-i \lambda}{l-\mu} B(l) A(\mu)+\frac{i \lambda}{l-\mu} B(\mu) A(l), \\
D(\mu) B(l) & =\frac{\mu-l-i \lambda}{\mu-l} B(l) D(\mu)+\frac{i \lambda}{\mu-l} B(\mu) D(l) .
\end{aligned}
$$


We finally put the following ansatz for the wavefunction:

$$
\left|k_{p_{1}} \cdots k_{p_{N}}\right\rangle=B\left(l_{1}\right) \cdots B\left(l_{M}\right)|\mathrm{vac}\rangle .
$$

By substituting the above into eq. (B.13) and using the commutation relations (B.21), (B.22), and (B.23), we obtain the following Bethe ansatz equations:

$$
\begin{aligned}
e^{i k_{j} L} & =-\prod_{l=1}^{N} \frac{k_{j}-k_{l}+i \lambda}{k_{j}-k_{l}-i \lambda} \prod_{\beta=1}^{M} \frac{k_{j}-l_{\beta}-i \lambda / 2}{k_{j}-l_{\beta}+i \lambda / 2}, \\
\prod_{j=1}^{N} \frac{l_{\alpha}-k_{j}-i \lambda / 2}{l_{\alpha}-k_{j}+i \lambda / 2} & =-\prod_{\beta=1}^{M} \frac{l_{\alpha}-l_{\beta}-i \lambda}{l_{\alpha}-l_{\beta}+i \lambda} .
\end{aligned}
$$

Finally, by taking the logarithm of the above equations, we obtain eqs. (3.2) and (3.3).

\section{Derivation of dressed energies}

For general $N$ and $M$, the ground state energy can be expressed as

$$
\begin{aligned}
E / L & =\frac{1}{L}\left(\sum_{j=1}^{N} k_{j}^{2}-\mu N-h M+\frac{h N}{2}\right) \\
& =\int_{-\Lambda}^{\Lambda} d k\left(k^{2}-\mu+h / 2\right) \rho(k)-h \int_{-\Omega}^{\Omega} d l \sigma(l),
\end{aligned}
$$

where $h$ is a magnetic field, and $\sigma(l)$ is the density of the isospin rapidities. From the Bethe ansatz equations, we can show

$$
\vec{\rho}(k, l)=\vec{\rho}_{0}(k, l)+K\left(k, l \mid k^{\prime}, l^{\prime}\right) \otimes \vec{\rho}\left(k^{\prime}, l^{\prime}\right)
$$

where

$$
\begin{aligned}
& \vec{\rho}(k, l)=(\rho(k), \sigma(l))^{T}, \\
& \vec{\rho}(k, l)=(1 /(2 \pi), 0)^{T}, \\
& K\left(k, l: k^{\prime} l^{\prime}\right)=\frac{1}{\pi}\left(\begin{array}{cc}
\frac{\lambda}{\lambda^{2}+\left(k-k^{\prime}\right)^{2}} & \frac{-\lambda / 2}{(\lambda / 2)^{2}+\left(k-l^{\prime}\right)^{2}} \\
\frac{\lambda / 2}{(\lambda / 2)^{2}+\left(l-k^{\prime}\right)^{2}} & \frac{-\lambda}{\lambda^{2}+\left(l-l^{\prime}\right)^{2}}
\end{array}\right),
\end{aligned}
$$

and $\otimes$ means the integral over the same variables.

We next introduce

$$
\vec{\varepsilon}_{0}(k, l)=\left(k^{2}-\mu-h / 2,0\right)^{T} .
$$

Then, the ground state energy can be rewritten as

$$
\begin{aligned}
E / L & =\vec{\varepsilon}_{0}^{T} \otimes \vec{\rho}, \\
& =\vec{\rho}_{0}^{T} \otimes \vec{\varepsilon},
\end{aligned}
$$

where $\vec{\varepsilon}=\left(\epsilon_{c}(k), \epsilon_{s}(l)\right)^{T}$ is the dressed energy. This satisfies

$$
\vec{\varepsilon}=\vec{\varepsilon}_{0}+K^{T} \otimes \vec{\varepsilon}
$$

which is nothing but eqs. (3.7) and (3.8). 


\section{Tomonaga-Luttinger liquid in two-component Bose gases}

Here, we consider the following Hamiltonian in two-component bosons,

$$
H=\int d x \sum_{\sigma}\left(\frac{\hbar^{2}}{2 m} \partial \Psi_{\sigma}^{\dagger} \partial \Psi_{\sigma}-\mu \Psi_{\sigma}^{\dagger} \Psi_{\sigma}+\frac{\lambda}{2}\left(\Psi_{\sigma}^{\dagger} \Psi_{\sigma}\right)^{2}\right)+\kappa \Psi_{1}^{\dagger} \Psi_{1} \Psi_{2}^{\dagger} \Psi_{2} .
$$

Here, we are interested in the miscible case, that is,

$$
\begin{aligned}
& \lambda>\kappa, \\
& \lambda>0 .
\end{aligned}
$$

In what follow, we consider the situation that two components have the same density, $\rho_{1}=\rho_{2}$. Then, we can apply the bosonization to this "bosonic" system. In the bosonization for bosons [27], we can use the following bosonization

$$
\begin{aligned}
& \Psi_{\sigma}^{\dagger}=\left(\rho_{0}-\frac{1}{\pi} \nabla \phi_{\sigma}\right)^{1 / 2}\left[e^{-i \theta_{\sigma}}+\sum_{p= \pm 1} e^{2 i p\left(\pi \rho_{0} x-\phi_{\sigma}\right)-i \theta_{\sigma}}\right] \\
& \rho_{\sigma}=\Psi_{\sigma}^{\dagger} \Psi_{\sigma}=\left(\rho_{0}-\frac{1}{\pi} \nabla \phi_{\sigma}\right)+\rho_{0} \sum_{p= \pm 1} e^{2 i p\left(\pi \rho_{0} x-\phi_{\sigma}\right)}
\end{aligned}
$$

where $\phi_{\sigma}$ and $\theta_{\sigma}$ are conjugate fields in the bosonization.

By applying the above dictionary to the our Hamiltonian, we have

$$
H \rightarrow \frac{1}{2 \pi} \int d x \sum_{\mu=\rho, \sigma}\left[u_{\mu} K_{\mu}\left(\nabla \theta_{\mu}\right)^{2}+\frac{u_{\mu}}{K_{\mu}}\left(\nabla \phi_{\mu}\right)^{2}\right]+2 \kappa \rho_{0}^{2} \int d x \cos 2 \sqrt{2} \phi_{\sigma},
$$

where we introduce

$$
\begin{aligned}
\phi_{\rho} & =\frac{1}{\sqrt{2}}\left(\phi_{1}+\phi_{2}\right), \\
\phi_{\sigma} & =\frac{1}{\sqrt{2}}\left(\phi_{1}-\phi_{2}\right),
\end{aligned}
$$

and the similar relations for $\theta$ fields. Here, $u_{\mu}$ is the velocity and $K_{\mu}$ is the so-called Luttinger parameter, and they are given by

$$
\begin{aligned}
K_{\rho} & =\frac{K}{\sqrt{1+\kappa K / \pi u}}, \\
K_{\sigma} & =\frac{K}{\sqrt{1-\kappa K / \pi u}}, \\
u_{\rho} & =u \sqrt{1+\kappa K / \pi u} \\
u_{\rho} & =u \sqrt{1-\kappa K / \pi u} \\
K & =\sqrt{\frac{\pi^{2} \rho_{0}}{\lambda M}} \\
u & =\sqrt{\frac{\lambda \rho_{0}}{M}} .
\end{aligned}
$$


As can be seen from the above bosonization Hamiltonian, if there is no coupling between up and down particles $(\kappa=0)$, the Hamiltonian is quadratic, which is called Tomonaga-Luttinger Hamiltonian.

In the presence of $\kappa$, we also have $\cos \phi_{\sigma}$, and therefore physics becomes non-trivial. To see what happens in the presence of this coupling, we can consider the renormalization group analysis, which is one of the most powerful approaches in one dimensional world. By applying the perturbative renormalization group in the above Hamiltonian, we have the following renormalization group equations [27],

$$
\begin{aligned}
\frac{d K_{\sigma}(l)}{d l} & =-\frac{\kappa_{12}^{2}(l) K_{\sigma}^{2}(l)}{2}, \\
\frac{d \kappa(l)}{d l} & =\left(2-2 K_{\sigma}(l)\right) \kappa(l) .
\end{aligned}
$$

We note that the above are essentially equivalent to renormalization group equations in the so-called BKT transition. We can easily check that $\cos \phi_{\sigma}$ is locked since $\kappa$ flows to strong coupling if $K_{\sigma}<1$ while $\cos \phi_{\sigma}$ is irrelevant if $K_{\sigma}>1$.

In a weakly-interacting bosonic system, we naturally expect that $K_{\sigma}>1$, and therefore the fixed point is the Tomonaga-Luttinger liquid phase. Namely, even if we consider the quantum fluctuations, we still expect that the low-energy excitations in the system are linear gapless.

Open Access. This article is distributed under the terms of the Creative Commons Attribution License (CC-BY 4.0), which permits any use, distribution and reproduction in any medium, provided the original author(s) and source are credited.

\section{References}

[1] A. Hanany and D. Tong, Vortices, instantons and branes, JHEP 07 (2003) 037 [hep-th/0306150] [INSPIRE].

[2] R. Auzzi, S. Bolognesi, J. Evslin, K. Konishi and A. Yung, Non-abelian superconductors: Vortices and confinement in $N=2$ SQCD, Nucl. Phys. B 673 (2003) 187 [hep-th/0307287] [INSPIRE].

[3] M. Eto, Y. Isozumi, M. Nitta, K. Ohashi and N. Sakai, Moduli space of non-abelian vortices, Phys. Rev. Lett. 96 (2006) 161601 [hep-th/0511088] [INSPIRE].

[4] M. Eto et al., Non-abelian vortices of higher winding numbers, Phys. Rev. D 74 (2006) 065021 [hep-th/0607070] [INSPIRE].

[5] M. Eto, Y. Isozumi, M. Nitta, K. Ohashi and N. Sakai, Solitons in the Higgs phase: the moduli matrix approach, J. Phys. A 39 (2006) R315 [hep-th/0602170] [INSPIRE].

[6] M. Shifman and A. Yung, Supersymmetric solitons and how they help us understand non-abelian gauge theories, Rev. Mod. Phys. 79 (2007) 1139 [hep-th/0703267] [INSPIRE].

[7] M. Shifman and A. Yung, Supersymmetric Solitons, Cambridge Monographs on Mathematical Physics, Cambridge University Press, Cambridge U.K. (2009).

[8] D. Tong, Quantum vortex strings: a review, Annals Phys. 324 (2009) 30 [arXiv:0809.5060] [INSPIRE]. 
[9] A.P. Balachandran, S. Digal and T. Matsuura, Semi-superfluid strings in high density QCD, Phys. Rev. D 73 (2006) 074009 [hep-ph/0509276] [InSPIRE].

[10] E. Nakano, M. Nitta and T. Matsuura, Non-abelian strings in high density QCD: zero modes and interactions, Phys. Rev. D 78 (2008) 045002 [arXiv:0708.4096] [INSPIRE].

[11] M. Eto and M. Nitta, Color Magnetic Flux Tubes in Dense QCD, Phys. Rev. D 80 (2009) 125007 [arXiv: 0907.1278] [INSPIRE].

[12] M. Eto, E. Nakano and M. Nitta, Effective world-sheet theory of color magnetic flux tubes in dense QCD, Phys. Rev. D 80 (2009) 125011 [arXiv:0908.4470] [INSPIRE].

[13] M. Eto, M. Nitta and N. Yamamoto, Instabilities of non-abelian vortices in dense QCD, Phys. Rev. Lett. 104 (2010) 161601 [arXiv:0912.1352] [INSPIRE].

[14] M. Eto, Y. Hirono, M. Nitta and S. Yasui, Vortices and other topological solitons in dense quark matter, PTEP 2014 (2014) 012D01 [arXiv: 1308.1535] [INSPIRE].

[15] A. Gorsky, M. Shifman and A. Yung, Confined magnetic monopoles in dense QCD, Phys. Rev. D 83 (2011) 085027 [arXiv:1101.1120] [INSPIRE].

[16] M. Eto, M. Nitta and N. Yamamoto, Confined monopoles induced by quantum effects in dense QCD, Phys. Rev. D 83 (2011) 085005 [arXiv:1101.2574] [INSPIRE].

[17] M. Shifman and A. Yung, Non-abelian string junctions as confined monopoles, Phys. Rev. D 70 (2004) 045004 [hep-th/0403149] [INSPIRE].

[18] A. Hanany and D. Tong, Vortex strings and four-dimensional gauge dynamics, JHEP 04 (2004) 066 [hep-th/0403158] [INSPIRE].

[19] S.R. Coleman, There are no Goldstone bosons in two dimensions, Commun. Math. Phys. 31 (1973) 259.

[20] N.D. Mermin and H. Wagner, Absence of ferromagnetism or antiferromagnetism in one-dimensional or two-dimensional isotropic Heisenberg models, Phys. Rev. Lett. 17 (1966) 1133 [INSPIRE].

[21] N. Dorey, T.J. Hollowood and D. Tong, The BPS spectra of gauge theories in two-dimensions and four-dimensions, JHEP 05 (1999) 006 [hep-th/9902134] [INSPIRE].

[22] H.B. Nielsen and S. Chadha, On how to count Goldstone bosons, Nucl. Phys. B 105 (1976) 445 [INSPIRE].

[23] H. Watanabe and H. Murayama, Unified description of Nambu-Goldstone bosons without Lorentz invariance, Phys. Rev. Lett. 108 (2012) 251602 [arXiv:1203.0609] [INSPIRE].

[24] Y. Hidaka, Counting rule for Nambu-Goldstone modes in nonrelativistic systems, Phys. Rev. Lett. 110 (2013) 091601 [arXiv: 1203.1494] [INSPIRE].

[25] D.S. Hall et al., Dynamics of component separation in a binary mixture of Bose-Einstein condensates, Phys. Rev. Lett. 81 (1998) 1539 [Erratum ibid. 81 (1998) 4531].

[26] H.J. Miesner et al., Observation of metastable states in spinor Bose-Einstein condensates, Phys. Rev. Lett. 62 (1989) 2228.

[27] T. Giamarchi, Quantum physics in one dimension, Oxford University Press, Oxford U.K. (2003).

[28] F. Essler et al., The one-dimensional Hubbard model, Cambridge University Press, Cambridge U.K. (2010). 
[29] M.A. Cazalilla et al., One dimensional bosons: from condensed matter systems to ultracold gases, Rev. Mod. Phys. 83 (2011) 1405.

[30] N.S. Manton, A remark on the scattering of BPS monopoles, Phys. Lett. B 110 (1982) 54 [INSPIRE].

[31] M. Eto, Y. Isozumi, M. Nitta, K. Ohashi and N. Sakai, Manifestly supersymmetric effective Lagrangians on BPS solitons, Phys. Rev. D 73 (2006) 125008 [hep-th/0602289] [INSPIRE].

[32] M. Kobayashi and M. Nitta, Kelvin modes as Nambu-Goldstone modes along superfluid vortices and relativistic strings: finite volume size effects, Prog. Theor. Exp. Phys. (2014) 021B01 [arXiv: 1307.6632] [INSPIRE].

[33] M. Gaudin, Un système à une dimension de fermions en interaction, Phys. Lett. A 24 (1967) 55 .

[34] C.-N. Yang, Some exact results for the many body problems in one dimension with repulsive delta function interaction, Phys. Rev. Lett. 19 (1967) 1312 [INSPIRE].

[35] T. Deguchi et al., Thermodynamics and excitations of the one-dimensional Hubbard model, Phys. Rep. 331 (2000) 197.

[36] Y.-Q. Li et al., Exact results of the ground state and excitation properties of a two-component interacting Bose system, Europhys. Lett. 61 (2003) 368.

[37] J.N. Fuchs et al., Spin waves in a one-dimensional spinor Bose gas, Phys. Rev. Lett. 95 (2005) 150402.

[38] E.H. Lieb and W. Liniger, Exact analysis of an interacting Bose gas. 1. The general solution and the ground state, Phys. Rev. 130 (1963) 1605 [InSPIRE].

[39] E.H. Lieb, Exact analysis of an interacting Bose gas. 2. The excitation spectrum, Phys. Rev. 130 (1963) 1616 [INSPIRE].

[40] E. Eisenberg and E.H. Lieb, Polarization of interacting bosons with spin, Phys. Rev. Lett. 89 (2002) 220403.

[41] M. Kobayashi and M. Nitta, Non-relativistic Nambu-Goldstone modes associated with spontaneously broken space-time and internal symmetries, to be published in Phys. Rev. Lett., arXiv:1402.6826 [INSPIRE].

[42] D.A. Takahashi and M. Nitta, Counting rule of Nambu-Goldstone modes for internal and spacetime symmetries: Bogoliubov theory approach, arXiv:1404.7696 [INSPIRE].

[43] M. Kobayashi and M. Nitta, Non-relativistic Nambu-Goldstone modes propagating along a skyrmion line, Phys. Rev. D 90 (2014) 025010 [arXiv:1403.4031] [INSPIRE]. 